\title{
PRODUCCIÓN Y CONSUMO DE CARNE DE CONEJO EN SANTA CATARINA/BRASIL
}

\author{
Andrei Bonamigo ${ }^{1}$ \\ https://orcid.org/0000-0002-6670-9755 \\ César Augustus Winck ${ }^{2}$ \\ https://orcid.org/0000-0002-9829-1824 \\ Recebido em: 9 jul. 2020 \\ Aceito em: 27 jul. 2020
}

\begin{abstract}
Como citar este artigo: BONAMIGO, Andrei; WINCK, César Augustus. PRODUCCIÓN Y CONSUMO DE CARNE DE CONEJO EN SANTA CATARINA/BRASIL. Revista Visão: Gestão Organizacional, Caçador, SC, Brasil, v. 9, n. 2, p. 73-86, jul./dez. 2020. Disponível em: http://dx.doi.org/10.33362/visao.v9i2.2318.
\end{abstract}

Resumen: El estudio se desarrolla sobre la cunicultura. Objetivo: realizar un sondaje sobre la producción y el consumo de carne de conejo. Tiene un planteamiento cuantitativo y cualitativo, se caracteriza como descriptivo y como levantamiento. Realizado con productores, cooperativa de cunicultores, comerciantes y cliente final, en la región del medio Oeste de Santa Catarina. Los resultados obtenidos evidencian que los factores limitadores de la actividad y del consumo de los productos de la cultura cunicular se relacionan a la organización de la actividad y a la interrelación de los actores de la cadena. Principales factores que den motivo para el consumo de esa carne: la curiosidad, el hecho de ser un alimento saludable y el sabor. Aspectos relacionados a la oportunidad, cultura y acceso justifican o no el consumo. El acceso al producto se da por medio de amigos o conocidos y directamente con productores. Los consumidores consideran difícil la preparación de ese alimento y, cuando lo hacen, eso sucede en comidas con amigos y familiares los fines de semana. Es necesario articular los diferentes segmentos de la cadena productiva cunicular para que se creen estrategias en red, fomentando así un sistema de producción competitivo para el agro-negocio cunicular catarinense. La actividad cunicular necesita ser incentivada en Santa Catarina, tanto en la producción como en el consumo, ya que existe potencial para la carne y sus derivados, pero es necesario reducir las limitaciones que la cadena productiva enfrenta en la actualidad.

Palabras-Clave: Agronegocio. Conejo. Cunicultura. Producción. Consumo.

PRODUÇÃO E CONSUMO DA CARNE DE COELHO EM SANTA CATARINA/BRASIL

Abstract: O estudo se descreve sobre a cultura, com o objetivo de realizar um diagnóstico sobre a produção e o consumo de carne de coelho. Para atingir esse objetivo, foi conduzido entrevistas com produtores, cooperativas de cooperativas, comerciantes e clientes finais, na região do meio oeste de Santa Catarina/Brasil. Os resultados obtidos evidenciam que os fatores limitadores da atividade e consumo de produtos da cultura cunícula se relacionam à organização da atividade e à inter-relação dos atores da cadeia de produção. Principais fatores que determinam o consumo da carne estão atrelados a curiosidade, alimento saudável e o sabor. Aspectos relacionados à oportunidade, hábito

\footnotetext{
${ }^{1}$ Doutor. Universidade Federal Fluminense - UFF. E-mail: andreibonamigo@gmail.com.

${ }^{2}$ Doutor. Universidade do Alto Vale do Rio do Peixe - UNIARP. E-mail: cesar.cepan@gmail.com.
} 
e acesso ao produto, foram fatores identificados como limitadores do consumo. O acesso ao produto ocorrer por meio de amigos ou contatos e diretamente com produtores, ou seja, informalmente. Os consumidores consideram difícil a preparação do alimento e este ocorre em momentos especiais e em finai de semana. Diante dos achados, pode-se constatar a necessidade de articular os diferentes segmentos da cadeia produtiva para estratégias de organização da cadeia produtiva, fomentando um sistema de produção competitivo para a negociação cunícula catarinense. Há oportunidade para incentivar e organizar a produção de coelhos em Santa Catarina, de modo a tornar uma cadeia de produção competitiva e sustentável economicamente.

Palavras-chave: Agronegócio. Coelho. Cunicultura. Produção. Consumo

\section{INTRODUÇÃO}

La producción de conejos, también llamada de cunicultura, es una actividad agropecuaria que ofrece al productor, además de perspectivas económicas, diversas oportunidades de aprovechamiento del animal y sus derivados. El conejo presenta posibilidades de comercialización, prácticamente en su totalidad, como: la carne, pelo, orina, entre otros. Este ramo del agro-negocio es poco aprovechado en Brasil, representando gran potencial de crecimiento (SANTOS, 2010; BONAMIGO, 2014). Como afirma Tvardovskas (2012), los conejos dan productos que pueden ser aplicados en actividades que van desde la producción de piezas artesanales a artículos de las industrias textiles y farmacéuticas.

La cunicultura se presenta como alternativa complementar en la producción agrícola familiar para Santa Catarina, ya que posibilita ganancias con la participación de la familia como mano de obra. El estudio tuvo como objetivo realizar un sondaje sobre la producción y consumo de carne de conejo en Santa Catarina (Brasil). Para el alcance del mismo, fueron definidos objetivos específicos, siendo ellos: identificar los factores que limitan la actividad cunicular, así como el destino de la producción; enumerar los motivos para el consumo y para el no consumo de la carne de conejo; identificar la forma de preparación de la carne, los canales de acceso y las ocasiones de preferencia para el consumo.

\section{LA ACTIVIDAD CUNICULAR}

En la nutrición humana, los alimentos oriundos de productos de origen animal son importantes fuentes de proteínas y de otros nutrientes. La carne de conejo se encuadra en esta realidad ya que es considerada más libre de grasa y más saludable, cuando comparada a las carnes bovinas, ovinas, y porcinas. Además de eso, es altamente digerible, sabrosa, con reducidas calorías, gorduras y colesterol y frecuentemente recomendada por nutricionistas en detrimento de las otras carnes (HERNADEZ et al, 2000).

La contribución de Gonzales (2006) apunta que las características de la carne de 
conejo, con relación a las demás especies analizadas en el estudio, presentan diversas ventajas con relación a las demás: por la baja tasa de gorduras, colesterol y elevado nivel de proteínas y fierro.

En su uso en dietas, la carne de conejo contribuye para una cantidad significativa de calcio, fósforo y vitaminas, siendo recomendada para niños, ancianos y especialmente para aquellos con colesterol elevado, riesgo de arterioesclerosis y enfermedades cardiovasculares (COLIN, 2010) y un producto alimentar saludable, de fácil digestión. (DALLE ZOTTE, 2000).

La carne de conejo es una fuente de vitaminas del complejo B (B2, B3, B5, B12), como relatado por Combes (2004). En los conejos, calidad de carcasa, cantidad y proporción de ácidos grasos, ácidos en la composición de la carne y del tejido adiposo son controlados (COBOS; CAMBERO; ORDONEZ, 1993). La composición química de la carne de conejo es variable especialmente en el tenor de gordura, para cada sección de la carcasa. (PLA; PASCUAL; ARINO; 2004).

\section{PRODUCCIÓN Y ABATE DE CONEJOS}

En el sistema de producción de conejos, es necesario considerar tres maneras de operación, cada uno de los cuales con su objetivo definido. Esos modos son: producción de conejo extensivo, se intensivo e intensivo. El primero se puede encajar en lo que se llama "Sistema Tradicional" y los dos últimos corresponden al Ilamado "Sistema Industrial" (LLEONART et al., 1980). El "Sistema Tradicional" también es conocido como prorrogado o "patio trasero", es caracterizado por ser, en general, de pequeña cantidad y limitados cuidados técnicos (GONZALEZ, 2006). Ya el "Sistema Industrial" enfoca la alta producción, dirigida a la producción en escala y al mercado previamente establecido de acuerdo con los requisitos de marcación y de abastecimiento demandada por el mercado. (LLEONART et al., 1980).

El conejo es una especie que presenta elevado rendimiento productivo en ritmo intenso, ya que en la fase de crecimiento alcanzan aumento de peso medio de 40 gramos por día, alcanzando la edad del abate 40 días después del desmame, cuando son usadas dietas debidamente balanceadas representando una conversión alimentar comparable a la del pollo comercial. (DE BLAS, 1984).

De acuerdo con Birchard y Sherding (1996), las hembras maduran sexualmente entre 4 a 8 meses. Machos maduran entre seis a diez meses de edad. Siendo que la gestación dura, aproximadamente, 30-33 días, factor éste que contribuye para los niveles de productividad presentada.

Generalmente, una coneja de buena genética, o sea con buenos índices zootécnicos, está lista para el apareamiento en hasta 16 semanas de edad, siendo que el factor de la raza del animal es una buena influencia en este proceso para la edad de madurez sexual, donde se 
puede citar a las razas: blanca de la Nueva Zelandia y los Californianos (MCNITT, et al., 1996).

La distribución de efectivos de conejos en Santa Catarina, de acuerdo con el IBGE (2012), vía subdivisiones por Meso-regiones, congrega diversos municipios de un área geográfica, con similitudes económicas y sociales. Al analizar la meso-región Oeste catarinense, se verifica que ésta representa 50,42\% (18.909 cabezas) del efectivo total de la Provincia de Santa Catarina (37.501 mil cabezas) (IBGE, 2012).

\section{MATERIAL Y MÉTODOS}

La investigación posee un abordaje que mezcla dados cuantitativos y cualitativos. Se caracteriza, con referencia a los objetivos, como descriptiva y con referencia a los procedimientos, como un levantamiento.

La investigación fue realizada con productores, cooperativa de cunicultores, comerciantes y cliente final, en la región del medio Oeste de la Provincia de Santa Catarina. Fueron 03 productores autónomos definidos por conveniencia y 01 productor cooperado, sugerido por la cooperativa en función de su representatividad en términos de producción, totalizando 04 productores. En la Cooperativa " $\mathrm{A}$ ", el encuestado fue el presidente de la misma. Con referencia a los comerciantes de productos de la cunicultura, fueron seleccionados 04 clientes propuestos por la Cooperativa " $A$ ", delante de la representatividad de los mismos para la Cooperativa.

Entrevistas medio estructuradas fueron usadas para la colecta de datos junto a los productores, cooperativa de cunicultores y comerciantes Las entrevistas fueron grabadas y después transcriptas para análisis e interpretación.

Con relación al consumidor final de carne, (mercado consumidor), fueron enviados 500 cuestionarios, resultando en 304 efectivamente respondidos y devueltos. La colecta de datos fue por medio de la Web 2.0, Google docs, usando un cuestionario medio estructurado desarrollado con el auxilio de software para tratamiento do dados estadísticos. El cuestionario fue enviado a todas las regiones geográficas de Santa Catarina, de manera estratificada, obteniéndose la siguiente representatividad en las respuestas válidas: Costa (5,92\%), Medio Oeste $(37,82 \%)$, Nordeste $(5,26 \%)$, Oeste $(34,21 \%)$, Altiplano Serrano $(8,22 \%)$ y Sur de la Provincia (8,55\%).

Después de la colecta de datos, los mismos fueron organizados para la presentación, análisis e interpretación. Los datos cuantitativos por su vez, fueron agrupados y lanzados en cuadros propios, siendo analizados en su contenido. 


\section{RESULTADOS Y DISCUSIÓN}

El presente capítulo presenta los resultados del estudio realizado, considerando la orden de los objetivos que fueron propuestos, o sea: factores limitadores en la actividad de la cunicultura, destino de la producción; motivos para el consumo; justificativa para el no consumo; forma de preparación de la carne de conejo; canales de acceso; ocasiones de preferencia para el consumo.

\section{PERCEPCIÓN DE LOS INVESTIGADOS SOBRE LA ACTIVIDAD DE LA CUNICULTURA}

Los resultados presentados en la secuencia emanan de la colecta de datos realizada junto a productores cooperados, productores autónomos, representante de cooperativa y comerciantes.

Los factores limitadores para el desarrollo de la actividad de la cunicultura de la Provincia de Santa Catarina, en la percepción de los productores autónomos y cooperados, se encuentran presentados en el Cuadro 1.

Cuadro 1 -Limitadores para el desarrollo de la actividad cunícola

\begin{tabular}{cc}
\hline Productor & Limitadores \\
\hline $\mathbf{P A}^{\mathbf{1}}$ & Falta de incentivo en el sector y requisitos presentados por los clientes (restaurantes y parrillas) \\
& $\begin{array}{l}\text { solicitando control sanitario e inspección de la carne; aplicación de apenas un medicamento } \\
\text { comercial, que utiliza el principio activo identificado como ivermectina }{ }^{1}\end{array}$ \\
$\mathbf{P A}^{2}, \mathbf{P A}^{3} \mathbf{E}$ & Falta de divulgación del producto, políticas públicas e incentivos gubernamentales. \\
$\mathbf{P C O}^{1}$ & \\
$\mathbf{P A}^{1}, \mathbf{P A}^{2}, \mathbf{P A}^{3} \mathbf{E}$ & La utilización de la ración comercial tiene costo elevado y periódicamente alimentan los \\
$\mathbf{P C O}^{1}$ & animales con legumbres y hortalizas (heno, pastaje, lechuga). \\
$\mathbf{P A}^{2}, \mathbf{P A}^{3}$ & Burocracia para adecuar las normas de abate y comercialización. \\
$\mathbf{P A}^{1}, \mathbf{P A}^{2}, \mathbf{P A}^{3} \mathbf{E}$ & Limitación operacional, o sea, bajas inversiones en el sistema de producción, \\
$\mathbf{P C O}^{1}$ & reaprovechamiento de equipamientos y materiales de construcciones antiguas de la propiedad. \\
$\mathbf{P A}^{2}$ & Dificultades para la venta de la producción se torna insegura en la cantidad de efectivos y \\
& matrices que se mantendrá en la propiedad. Bajo valor recibido por la carne y dificultad para \\
& aumentar su precio, donde el comprador lo establece. \\
\hline
\end{tabular}

Leyenda: PA - Productor autónomo; PCO - Productor cooperado.

Los factores limitadores, en la percepción del representante de la cooperativa son el acceso a los insumos, el proceso de producción/transformación, el destino de la producción y los medios de comercialización. Sobre las causas del bajo efectivo en la cultura cunicular en la Provincia de Santa Catarina, el representante mencionó aspectos relacionados a la valorización del producto, costos de producción, tecnologías usadas en la producción y transformación.

Se verifica diversidad en los factores limitadores de la actividad, que no se caracteriza como un nuevo concepto, sino una unión de diversos conceptos de economía, administración, marketing ya fundamentados, que se relacionan de forma dificultosa por la independencia y bajo relacionamiento entre los eslabones, que contribuyen para desorientar el objetivo global 
de la cadena de valor.

El reflejo de este escenario rescata la importancia de la integración de los eslabones en la búsqueda de ventaja competitiva y agregación de valor en la cadena de valores, con ese objetivo el eslabón en sí es el reflejo de la maximización del valor global generado, se tornan fundamentales para contribuir en la reducción de los limitadores apuntados.

En la secuencia, se presenta en el Cuadro 2, la caracterización de los establecimientos comerciales investigados.

Cuadro 2 - Caracterización de los establecimientos comerciales investigados

\begin{tabular}{|c|c|c|c|c|c|}
\hline Denominación & Ramo comercial & $\begin{array}{c}\text { Número } \\
\text { Sucursales }\end{array}$ & $\begin{array}{c}\text { Función del } \\
\text { Contacto }\end{array}$ & $\begin{array}{c}\text { Demanda } \\
\text { media } \\
\text { mensual por } \\
\text { sucursal } \\
\end{array}$ & $\begin{array}{c}\text { Tiempo que } \\
\text { comercializa } \\
\text { productos de La } \\
\text { cultura cunicular }\end{array}$ \\
\hline $\mathrm{CO1}$ & $\begin{array}{c}\text { Red de supermercados } \\
01\end{array}$ & 3 & $\begin{array}{l}\text { Coordinador de } \\
\text { carnicería }\end{array}$ & $68 \mathrm{~kg}$ & 3 años \\
\hline $\mathrm{CO2}$ & $\begin{array}{c}\text { Red } \\
\text { de supermercados } 02\end{array}$ & 4 & $\begin{array}{l}\text { Coordinador } \\
\text { carnicería }\end{array}$ & $70 \mathrm{~kg}$ & 2 años \\
\hline $\mathrm{CO3}$ & Restaurante & 1 & Propietaria & $58 \mathrm{~kg}$ & 6 años \\
\hline $\mathrm{CO4}$ & Restaurante con hotel & 1 & Socia/Gerente & $20 \mathrm{~kg}$ & 2 años \\
\hline
\end{tabular}

La percepción de los comerciantes entrevistados sobre las dificultades para la comercialización de productos de la cultura cunicular, constan en el Cuadro 3, presentados a seguir.

Cuadro 3- Dificultades para comercialización comentadas por los comerciantes

\begin{tabular}{|c|c|c|c|}
\hline Denominación & Dificultades & $\begin{array}{c}\text { Público consumidor } \\
\text { (edad) }\end{array}$ & Aspectos destacados \\
\hline $\mathrm{COl}$ & $\begin{array}{l}\text { Falta de divulgación, } \\
\text { productos sin } \\
\text { mejoramiento }\end{array}$ & Entre 40 a 60 años & $\begin{array}{l}\text { La mayoría de los consumidores son } \\
\text { personas de origen y/o que viven en } \\
\text { el medio rural }\end{array}$ \\
\hline $\mathrm{C} 02$ & $\begin{array}{l}\text { Falta del producto y } \\
\text { productos alternativos }\end{array}$ & Entre 40 a 50 años & $\begin{array}{c}\text { Consumo por eventualidades } \\
\text { "quebrar el ritmo de los } \\
\text { tradicionales" }\end{array}$ \\
\hline $\mathrm{C03}$ & $\begin{array}{l}\text { Divulgación de la } \\
\text { carne e incentivo al } \\
\text { consumo }\end{array}$ & $\begin{array}{c}\text { Entre } 50 \text { a } 60 \text { años. En torno } \\
\text { de } 75 \% \text { del consumo es por } \\
\text { público masculino }\end{array}$ & $\begin{array}{l}\text { Presentación y Demonstración de } \\
\text { nuevos platos }\end{array}$ \\
\hline $\mathrm{CO4}$ & $\begin{array}{l}\text { Divulgación de las } \\
\text { características } \\
\text { alimentares de la carne }\end{array}$ & Entre 40 a 50 años & $\begin{array}{l}\text { Presentar productos oriundos de la } \\
\text { carne en ferias y acontecimientos } \\
\text { gastronómicos }\end{array}$ \\
\hline
\end{tabular}

Delante de los datos constantes en el Cuadro 5, es posible identificar que la franja de edad en la que prevalece el público consumidor de carne de conejo, es de 40 a 60 años. El comerciante C03 destacó que, aproximadamente $75 \%$ de los consumidores de carne de conejo son representados por el público masculino. A partir de datos levantados, se verificó que las dificultades para la comercialización/consumo de carne están unidas a la falta de divulgación y elaboración del producto. 
Los comerciantes entrevistados declararon algunas características del público consumidor, el hecho de que, son personas principalmente de origen rural o que viven en el medio rural, de que muchas personas optan por el consumo de carne de conejo como factor de diversificación de los tipos de carne que consumen tradicionalmente, y también que, la innovación en términos de culinaria sería un atractivo al consumo. De acuerdo con el entrevistado C03 la necesidad de oferta de nuevos platos, a partir de la carne de conejo, es un factor que limita la promoción del consumo, destaca también que el proveedor de ese tipo de carne podría ser un medio para suprimir esa necesidad. Ya para C04 la presentación de los productos de la cultura cunicular en ferias y acontecimientos gastronómicos podría levantar el consumo de la carne.

A partir de las contribuciones del sector de comercialización de la carne de la cultura cunicular, eslabón que está más próximo del consumidor final, deja en evidencia la oportunidad para una adecuación del valor para el consumidor. Nickels y Wood (1999) relatan que es de suma importancia que la organización mantenga una orientación para el cliente e investigue su visión de la adecuación del valor, antes de realizar estrategias de precios dirigidas a nuevos clientes y al fortalecimiento de los clientes ya existentes.

Con las informaciones obtenidas, se identificó la diversidad de los destinos de los animales vivos y abatidos, así como el de los menudos, en el segundo caso. En el Cuadro 4 se puede observar el destino de la producción.

Cuadro 4. Destino de los animales vivos, in natura y menudos

\begin{tabular}{|c|c|c|c|}
\hline Referencia & $\begin{array}{c}\text { Forma de } \\
\text { venta }\end{array}$ & $\begin{array}{c}\text { Destino de los } \\
\text { menudos }\end{array}$ & Principales Clientes \\
\hline $\mathrm{CO}$ & Cortes & Subproductos* & $\begin{array}{l}\text { Hoteles, Mercados, tiendas de carne, parrillas, restaurantes, } \\
\text { acontecimientos regionales. }\end{array}$ \\
\hline PCOl & Vivo & Subproductos* & Cooperado \\
\hline PAl & In natura & Compostaje & Comunidad por conveniencia, parrillas y restaurantes \\
\hline PA2 & In natura & Compostaje & Comunidad por conveniencia, parrillas y restaurantes \\
\hline PA3 & In natura & Compostaje & Comunidad por conveniencia \\
\hline \multicolumn{4}{|c|}{ *Considera-se cualquier mejoramiento que genere valor comercial para os menudos. } \\
\hline
\end{tabular}

Legenda: PA: Productor autónomo; PCO: Produtor cooperado; CO: representante da Cooperativa

Se verifica que los productores cooperados presentan mejorías en los menudos de los animales. Ya los productores autónomos no demuestran procesos de beneficios, apenas compostaje de estos.

Los animales listos para el abate son encaminados para la cooperativa y después del abate y beneficio del producto, es hecho el direccionamiento para la comercialización por la misma. Ya en el caso de los productores autónomos el abate es realizado dentro de las propias propiedades rurales y enseguida comercializado, por conveniencia, a la comunidad y a pequeños restaurantes y parrillas.

Es posible observar que los productores cooperados direccionan los menudos de los 
animales al procesamiento de manera a agregar valor a los "residuos" generados en el proceso del abate. Ya los productores autónomos practican el descarte de los "residuos" por medio de compostaje, lo que representa un factor limitador para la maximización de la actividad y sustentabilidad económica del agricultor.

Los resultados presentados en la secuencia, reflejan las opiniones de 304 consumidores finales que participaron de la investigación, respondiendo al interrogatorio electrónico. El perfil de los consumidores investigados se caracteriza, principalmente como siendo del género femenino (58\%), con menos de 25 años (41\%). Cincuenta y ocho por ciento de los investigados son solteros.

Vía investigación aplicada a la población de Santa Catarina, pueden conocerse las percepciones del mercado consumidor de Santa Catarina sobre los productos de la cultura cunicular. Entre los entrevistados se destaca que el $58 \%$ de los investigados ya consumieron carne de conejo y los demás $42 \%$ no habían consumido el producto. El análisis de ese limitador en el eslabón del consumidor está de acuerdo con las indicaciones de Carvalho (2009) que informa que la carne de conejo continúa a ser un producto poco requerido, siendo su consumidor un nicho de mercado.

Sobre la justificativa para el consumo de carne, se obtuvieron diferentes respuestas, destacando los factores presentados en la Figura 1.

Figura 1. Factores que llevan al consumo de carne de conejo

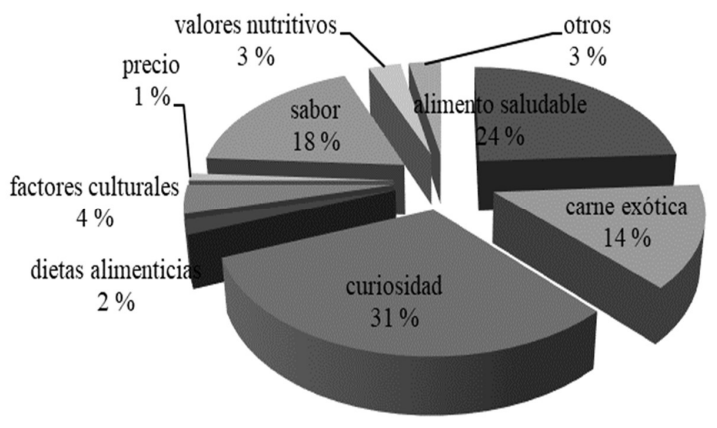

El factor curiosidad (31\%) fue la principal justificativa de consumo, a continuación, por ser un alimento saludable (24\%), por motivos de sabor (18\%), por ser una carne exótica (14\%), por factores culturales (4\%) y por precio (1\%). Las justificativas para el no consumo de carne de conejo, destacadas por los investigados, pueden verificarse en la Figura 2. 
Figura 2. Justificativa para el no consumo de carne de conejo

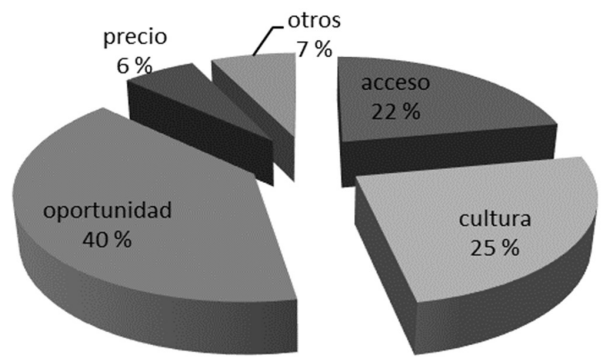

El principal motivo para el no consumo de carne de conejo, fue la cuestión de la oportunidad para acceder al producto (40\%), seguido en orden decreciente por cultura (25\%) y acceso (22\%). El factor precio fue el motivo citado por apenas $6 \%$ de los investigados. Los resultados pueden estar indicando que el consumo de carne de conejo está directamente relacionado a aspectos individuales y personales de cada consumidor.

Al interrogar a los respondientes de la investigación sobre el conocimiento de la carne de conejo como una opción alimentar saludable, se verificó que la mayoría no tenía conocimiento $(56 \%)$ y el restante $(44 \%)$ reconoció ser una opción alimentar saludable.

Los datos marcan que los entrevistados se limitan a los conocimientos sobre la caracterización del producto, factor que contribuyó para promover interés y la búsqueda por el consumo de la carne de conejo. Tejon (2009) afirman que el marketing es una herramienta apropiada para reaccionar a las tendencias de cambios percibidos en los negocios agropecuarios, a ejemplo del mayor énfasis a los productos con elevado valor agregado. Sobre la preparación de la carne de conejo se obtuvieron los porcentuales presentados en la Figura 3.

Figura 3. Preparación de la carne de conejo

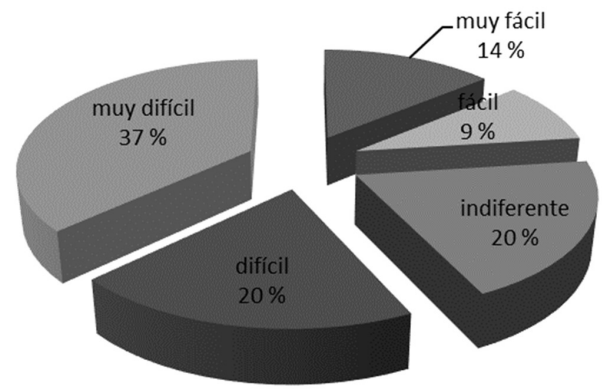


La mayoría que respondió, o sea, 57\% destacó como difícil a muy difícil la preparación de la carne de conejo, ya $23 \%$ consideran de fácil a muy fácil. La adquisición de alimentos, así como de otros bienes, se torna parte de un sistema de reputación, involucrando juzgamientos sobre buen gusto, en los cuales las diferentes clases sociales eligen algunos ítems con relación a otros delante de las demás variaciones de influencia en la elección (BOURDIEU, 1984).

Con referencia a los factores relacionados al acceso se dejó en evidencia una falta de padrón en los canales de obtención del producto, de acuerdo con lo que es posible observar en la Figura 4.

Figura 4. Canales de acceso a la carne de conejo

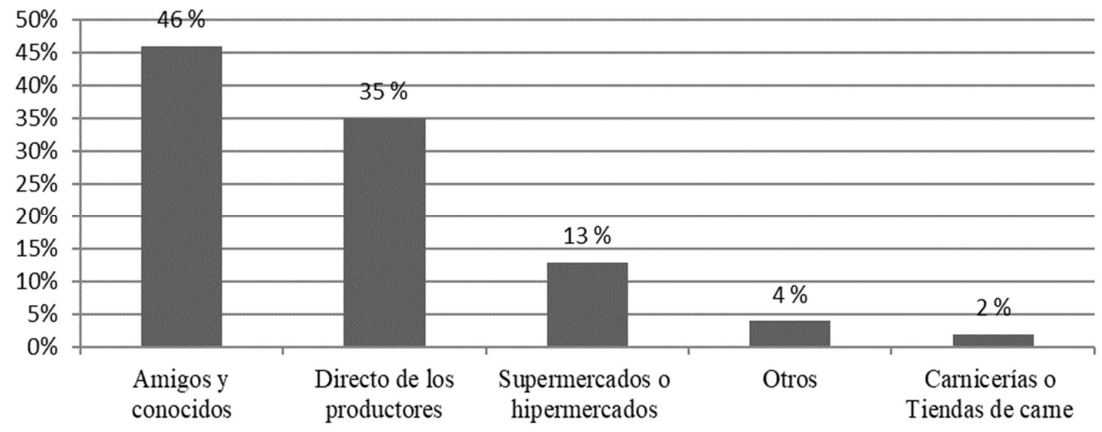

El acceso al producto representa el canal de acceso al mismo. El canal de acceso, con predominancia (46\%) en la respuesta, fue aquel que ocurre por intermedio de amigos o conocidos, en la secuencia, por medio directo a los productores (35\%), enseguida por supermercados o hipermercados (13\%), otros (4\%) y por último en carnicerías o tiendas de carnes (2\%).

Al preguntarle a los entrevistados en qué ocasiones prefieren consumir carne de conejo, apenas 150 presentaron respuestas, de acuerdo con la distribución presentada en el cuadro 5.

Cuadro 5. Ocasiones de preferencia para consumo de carne de conejo

\begin{tabular}{l|c|c}
\hline \multicolumn{1}{c|}{ Ocasiones de consumo } & $\mathbf{N}^{\circ}$ & $\%$ \\
\hline En comidas con los amigos y familiares los fines de semana & 42 & 14 \\
\hline Esporádicamente en cualquier día de la semana & 39 & 13 \\
\hline Cuando almuerza o cena fuera (restaurantes, parrillas y otros) y en acontecimientos públicos & 37 & 12 \\
\hline Cuando recibe visitas especiales en su casa, en fechas especiales (cumpleaños, fiestas) & 32 & 10 \\
\hline No respondieron & 154 & 51 \\
\hline
\end{tabular}


Se verifica que las ocasiones de consumo de la carne son diversificadas, siendo que de los 150 respondientes de esta cuestión, 14\% acostumbran a consumir la carne de conejo con amigos y familiares los fines de semana, 13\% tienen el hábito de consumo esporádico, cualquier día de la semana. El consumo cuando almuerza o cena fuera de casa (restaurante, parrillas, etc.) y en acontecimientos públicos, está representado por el 12\%. La minoría que respondió (10\%), afirma que consume la carne de conejo cuando recibe visitas especiales en su casa, en fechas especiales (cumpleaños, fiestas).

Se nota que la carne tiende a ser apreciada en momentos especiales, atípico de las carnes tradicionalmente consumidas cotidianamente por la población catarinense. Una ventaja delante de las carnes tradicionales es que el consumidor no asocia la carne de conejo a cualquier patología, como sucede con otros animales, y eso debe ser aprovechado para alimentar su consumo (CARVALHO, 2009). Es posible verificar que los momentos para consumo de la carne suceden en situaciones que favorecen al consumidor, su preparación, tal como en finales de semana o por medio de la disposición de platos de la carne, por otras personas.

\section{CONSIDERACIONES FINALES}

El presente estudio fue desarrollado con el objetivo de realizar un sondaje sobre la producción y consumo de carne de conejo en Santa Catarina. Para tanto, fue realizado un estudio teórico que tocó temas como el consumo de carne en Santa Catarina; la actividad cunicular; la producción y abate de Conejos; la cadena de valor y, agregación de valor. Fueron investigados productores, representante de cooperativa, comerciantes y público consumidor, usando entrevistas y la aplicación de cuestionarios.

Los resultados obtenidos auxilian en la identificación de la situación de producción y del consumo de la carne de conejo en Santa Catarina, dejando en evidencia que los factores limitadores de la actividad y del consumo de los productos de la cultura cunicular se relacionan a la organización de la actividad y de la interrelación de los actores de la cadena, con dificultades principalmente relacionadas con la falta de incentivo, control sanitario, inspección de la carne, la falta de divulgación del producto, costo elevado de la ración comercial, limitación operacional, acceso a los insumos, procesos de producción/transformación, destino de producción y medios de comercialización.

Con referencia al destino de la producción, se constató la existencia de diversidad para animales vivos y abatidos y también para menudos. Los productores cooperados encaminan los animales para el abate y para su aprovechamiento junto a la Cooperativa y direccionan los menudos al procesamiento, con el interés de agregar valor. Los productores autónomos los abaten en sus propiedades y los comercializan en la comunidad, y con los residuos preparan compostaje. 
Se verifica que el cunicultor presenta la necesidad de organización del trabajo, desarrollar relaciones para asegurar volumen de venta de animales, sistematizando su actividad con relación al mercado y a la aplicación de nuevas tecnologías de producción como inseminaciones y herramientas de gestión de la actividad.

Como limitación del estudio se verificaron los pocos estudios científicos sobre la actividad e investigaciones en el área de la cunicultura relacionada a la cadena productiva. Y, así mismo, los datos secundarios disponibles para la investigación son pocos y desfasados. Aunque cuidados metodológicos hayan sido adoptados, la muestra de sujetos investigados fue decurrente de direccionamiento del link para sujetos pertenecientes al network del investigador, lo que no, necesariamente, retrata un perfil de respondientes que sean cocineros o que posean el hábito de ir a hacer compras para adquirir carne para preparar en sus cocinas. Deteniéndonos en ese limitador, se entiende como oportunidad para futuras investigaciones, replicar el cuestionario para restaurantes, cocinas industriales y otros establecimientos promotores de acontecimientos y que estén acostumbrados a preparar platos diferenciados.

Se presenta como oportunidad para futuros estudios el profundizar en el área de prácticas de conglomerados inclinados a la actividad de la cunicultura, por medio de la replicación de este estudio para atender al público objetivo, que será el potencial consumidor - restaurantes, casas de carnes, hospitales, escuelas, hogares de ancianos, entre otros; investigar la relación entre actores de la cadena de valor de manera de promover la organización de la cadena productiva; mecanismos para promover la divulgación y agregación de valor de los productos originarios de la carne, delante del mercado consumidor; estudios para el desarrollo de subproductos a partir de la carne; desarrollo de estudios cuantitativos sobre la influencia de los limitadores del consumo de la carne de la cultura cunicular en Santa Catarina y en otras regiones de Brasil.

\section{AGRADECIMIENTOS}

A los productores de conejos, a la Cooperativa de cunicultores de la ciudad de Ouro/SC y región y a los establecimientos comerciales, por viabilizar los datos estadísticos. Agradecimiento al Fondo de Apoyo al Mantenimiento y al Desarrollo de la Educación Superior - FUMDES, pro contribuir con el financiamiento del estudio.

\section{REFERÊNCIAS}

BIRCHARD, S. J. et al. Manual clínico de pequeñas especies/Saunders manual of small animal practice. McGraw-Hill Interamericana, 1996.

BONAMIGO, A. Potencialidades e limitações na produção e consumo da carne cunícula em Santa Catarina. 2014. 132f. [Dissertação de Mestrado]. Universidade do Oeste de Santa 
Catarina, Chapecó, 2014.

BOURDIEU, P. Distinction: A social critique of the judgement of taste. Harvard university press, 1984.

CARVALHO, R. C. de. Caracterização da produção cunícula nas regiões De Trás-os-Montes, Minho e Galiza. 2009. 139f. [Dissertação de Mestrado]. Universidade de Tras-os-Montes e Alto Douro, Vila Real. 2009.

COBOS, A. et al. Effect of fat-enriched diets on rabbit meat fatty acid composition. Journal of the Science of Food and Agriculture, v. 62, n. 1, p. 83-88, 1993.

COLIN, M. La cuniculture chinoise. World Rabbit Science, v. 3, n. 3, p. 133-140, 2010.

DALLE ZOTTE, A. Main factors influencing the rabbit carcass and meat quality. In: Proc.: 7th World Rabbit Congress. 2000. p. 4-7.

DE BLAS, C. Alimentación del conejo, 2da Edición ed. Espano Andina, p. 43-56, 1984.

GONZÁLEZ REDONDO, P. Proposal of a nest box for the reproduction of wild rabbits (Oryctolagus cuniculus) in cages. World Rabbit Science, 14, 115-121, 2006.

HERNÁNDEZ, P., Pla, M., OLIVER, M. A., BLASCO, A. Relationships between meat quality measurements in rabbits fed with three diets of different fat type and content. Meat Science, v. 55, n. 4 , p. $379-384,2000$.

IBGE, Instituto Brasileiro de Geografia e Estatística. 2012. Censos. Consultado 01 de junio de 2019. Disponible en:http://www.ibge.gov.br/home/estatistica/indicadores/agropecuaria/producaoagropecuari a/

LLEONART, F., CAMPO, J. L., VALLS, R., CASTELLÓ, J. A., COSTA, P., PONTES, M. Tratado de cunicultura. Anatomía y fisiología del aparato digestivo. Barcelona: Real Escuela Oficial y Superior de Avicultura, v. 1, p. 61-84, 1980.

MCNITT, J. I.; LUKEFAHR, S. D. Genetic and environmental parameters for postweaning growth traits of rabbits using an animal model. In: World Rabbit Congress. 1996. V. 6, p. 325329.

NICKELS, W. G.; WOOD, M. B. Marketing: relacionamentos, qualidade, valor. Itc, 1999.

PLA, M.; PASCUAL, M.; ARIÑO, B. Protein, fat and moisture content of retail cuts of rabbit meat evaluated with the nirs methodology. World Rabbit Science, v. 12, n. 3, p. 149-158, 2010.

SANTOS, F. B. Cunicultura: análise de viabilidade de gerar uma empresa voltada para criação de 500 coelhos por mês em Feira de Santana, Bahia. 2010. [Monografia de Bacharelado]. Feira de Santana: Universidade Estadual de Feira de Santana. Consultado 02 de marzo de 
2014.

TEJON, José Luiz. Marketing \& agronegócio: a nova gestão: diálogo com a sociedade. Pearson Pretice Hall, 2009.

TVARDOVSKAS. L. S. Coelho. Consultado 11 de marzo de 2020. Disponible en: http://revistagloborural.globo.com/GloboRural/0,6993,EEC1411764-4530,00.html. 